\title{
Article \\ Influence of Altitude and Image Overlap on Minimum Mapping Size of Chemical in Non-Destructive Trace Detection Using Hyperspectral Remote Sensing
}

\author{
Siddharth Chaudhary*(D), Sarawut Ninsawat (D) and Tai Nakamura (D) \\ Remote Sensing and GIS, School of Engineering and Technology, Asian Institute of Technology, Klong Luang, \\ Pathum Thani 12120, Thailand; sarawutn@ait.ac.th (S.N.); nakamura.tai@rs.tus.ac.jp (T.N.) \\ * Correspondence: chaudharysiddharth@outlook.com
}

Citation: Chaudhary, S.; Ninsawat, S.; Nakamura, T. Influence of Altitude and Image Overlap on Minimum Mapping Size of Chemical in Non-Destructive Trace Detection Using Hyperspectral Remote Sensing. Appl. Sci. 2021, 11, 2586. https:// doi.org/10.3390/app11062586

Academic Editor: Byoung-Kwan Cho

Received: 10 December 2020

Accepted: 9 March 2021

Published: 14 March 2021

Publisher's Note: MDPI stays neutral with regard to jurisdictional claims in published maps and institutional affiliations.

Copyright: (c) 2021 by the authors. Licensee MDPI, Basel, Switzerland. This article is an open access article distributed under the terms and conditions of the Creative Commons Attribution (CC BY) license (https:// creativecommons.org/licenses/by/ $4.0 /)$.

\begin{abstract}
The increasing threat of explosives is a serious issue affecting socio-economy of many countries at multiple levels, such as public security, unused arable land, closing of trade routes, isolation of villages, and these act as a hindrance in the development of the country. Activities using explosives have increased in the last two decades making it a global threat that is challenging humanity. In this study, different chemicals such as Ammonium Nitrate (AN), Trinitrotoluene (TNT) and C4 along with soil as the background material were used for trace detection. The aim of this study was to determine an altitude for the sensor and to identify the minimum mapping size of the chemical at which the model can achieve $70 \%$ accuracy. To determine the altitude and minimum size of the chemical that can be detected in the acceptable range of accuracy, several experiments were performed in real ground conditions. This study focuses on the applicability of the proposed method in the real world. In the first set of experiments, the altitude of the sensor was varied from $40 \mathrm{~cm}$ to $150 \mathrm{~cm}$ and the accuracy of the model was determined. From the analysis, it was found that the model achieved more than $75 \%$ accuracy at an altitude of $90 \mathrm{~cm}$ with an image overlap of $70 \%$. In the second set of experiments, the minimum size of chemical sample was varied from $0.25 \mathrm{~cm}$ to $1 \mathrm{~cm}$. The accuracy of the model was more than $70 \%$ when the minimum sample size was $0.5 \mathrm{~cm}$ or greater. For various altitude determined, the speed of the vehicle was calculated. Therefore, to implement hyperspectral imaging system on the unarmed vehicle for real application, the suggested altitude and speed of the sensor should be around $90 \mathrm{~cm}$ and $10.5 \mathrm{~cm} / \mathrm{s}$ at which detection limit would be equal or more than $0.5 \mathrm{~cm}$ with accuracy greater than $70 \%$.
\end{abstract}

Keywords: hyperspectral imaging; trace detection; minimum mapping

\section{Introduction}

Activities involving explosives have increased in the last two decades and leading to a global threat which is challenging the humanity. Many terrorist attacks use a special type of bomb known as Improvised Explosive Devices (IED) in which the explosives are stored inside metal containers. The explosives are made up of chemical compounds which can create high impact even if used in less quantities. IEDs can be grouped as military, commercial and homemade on the basis of materials used for manufacturing them. Homemade explosive mixtures can be prepared by mixing inorganic energetic oxidant salts (Ammonium nitrate, Potassium nitrate, Potassium chlorate) with fuel like petrol, diesel, charcoal etc. [1]. The oxidant salts mentioned above are easily available in market in the form of fertilizers. Ammonium nitrate (AN) is one of the most commonly used fertilizers for agricultural purpose and combination of this with fuel can result in Ammonium Nitrate Fuel Oil (ANFO), dynamites that are explosive compounds. Similarly, Potassium Nitrate is a widely used fertilizer, but it is also used for manufacturing firecrackers by mixing it with black powder. Chemicals like ammonium nitrate, tri nitro toluene, $\mathrm{C} 4$ are used as explosive material. Ammonium nitrate is one of most common fertilizer used for 
agriculture, but a mixture of ammonium nitrate and fuel oil, in the ratio of 94:6, results in an explosive which is used widely. This type of bomb is gaining a lot of popularity as it can be manufactured using commonly available material at home [1-7]. In recent years, across the globe some major terrorist attacks were noticed in Pakistan, Brussels, Nigeria (2016), Paris (2015), Boston (2013) in which IED were widely used. IED is becoming an ideal weapon for spreading terrorism due to its special features like ease of manufacture at low cost and it can be designed in various sizes and forms making it difficult to be detected. Large number of conflicts are taking place across the world, particularly in a number of war zones. This is a serious issue that is affecting socio-economy of many countries like public security, unused arable land, closing of trade routes, isolation of villages. Such problems act as a hindrance in the development of the country. These problems motivate the government and research community to develop techniques for fast and accurate detection of explosives. Trace detection of explosives are usually achieved using several methods like electrochemical sensors, bio sensors and optical sensors. Most of these methods involves heavy, non-portable and complex instruments due to which analysis is not performed at the crime location or bomb sites making them not suitable for field applications $[8,9]$. Due to this forensic science and defense scientist are interested in developing detection technology which is non-destructive, portable with high sensitivity and selectivity, has ability to identify traces of chemicals in large background [10,11].

The above-mentioned requirements can be addressed using hyperspectral imaging system (HSI) which is relatively new technique based on hyperspectral imaging and visible near infrared (VSNIR) spectroscopy. Red Green Blue (RGB) devices give a valuable insight into the limitations of colour discrimination with a low number of photoreceptors, as the principles involved in the interpretation of photoreceptor signals in humans also apply to RGB camera responses. Whereas, the hyperspectral camera architecture provides means to explore other important aspects of colour vision like the perception of certain types of camouflage and colour constancy where multiple, narrow-band sensors increase resolution [12]. HSI has been used in many fields, such as food, agriculture, medical and target detection because of its unique combination of spectroscopy and imaging properties and characteristics like non-destructive, robust nature. HSI generates a hyperspectral cube in which $X Y$ represents the spatial information and $Z$ represents the wavelength. Scanning the samples and recording data for a wide area in less time is a major advantage of HSI over other vibrational spectroscopy technique. This technique gives the ability to remotely identify the composition of each pixel of the generated image [13]. The fast, non-invasive, non-destructive and contactless characteristic of HSI makes it a promising standoff trace detection tool as it provides remote and real-time measurements.

A key issue for identifying traces of chemical in the hyperspectral image from remotely sensed data is the identification of the minimum mapping size of the pure chemical. The accuracy of the model depends on several factors of like altitude of the sensor, velocity of the vehicle and the minimum size of the traces of the target chemical to be identified. A classified image, created for trace detection of chemical from classification model data, are presented using a minimum size of the chemical that can be mapped. It is defined as "the smallest size of the trace of the chemical that cab be mapped as a discrete entity".

Determining the minimum size of the pure chemical for trace detection of chemical in hyperspectral image is an important consideration due to the mix pixel problem in which a pixel can be dominated by the background material. Due to this limitation, it is often noticed that the performance of the algorithms used to process the hyperspectral data gets affected. The size of the chemical and resolution of the sensor can lead to challenging issue like mixed pixel [14]. If the quantity of the trace of the chemical is less than the background material in the pixel in that case mix pixel problem would arise. Irrespective of the spatial resolution of the sensor, in natural environmental conditions the spectral signature for a nominal pixel is invariably a mixture of the signatures of the background or clutter materials present in the field of view of the sensor [15]. This results in identification of pixels which contain mixture of chemical and background material, especially on the 
edges of the chemical, which reduces the overall accuracy. Very few articles have been published on determining minimum size of the trace of chemical to be identified and mixed pixel problem at a spatial resolution less than a centimeter. An inappropriate size of minimum mapping size of chemical results into "salt and pepper" effect where many single pixels of a target chemical that are interspersed with contiguous areas of other background material. Individual pixels are not spectrally pure because diffuse directional reflectance from neighboring pixels contribute to a given pixel's spectral signature. Such problem can be resolved by using appropriate size of minimum mapping size of chemical. Whereas, if the minimum mapping size of chemical is small then problem of mixed pixel problem is reduced as only pure pixels containing chemicals will be identified. Traces of chemicals that are sparse and fragmented can be considerably misrepresented in the final map when increasing minimum mapping size of chemical, while the classes that occupy a large percentage of map area tend to become more dominant. Given the preceding information, the choice of an appropriate MMS is clearly important. The suggested mapping size should provide as much information as possible at a minimum economic cost, without losing the necessary spatial information [16]. Hence, both precision and cost are factors that can have an effect on the final selection of the minimum mapping size of the chemical, but ideally, the suggested size falls within the constraints of the economics.

\section{Trade-Offs of Drone Operations}

In trace detection of chemical using hyperspectral sensors one of the major challenges is to determine the suitable parameter for the vehicle and the sensor, as shown in Figure 1. The goal of the developed technique is to identify the minimum size of the chemical without increasing the cost of capturing time, and image processing time excessively. To find the suitable minimum mapping size of the chemical, the system operator can select parameters like altitude, image overlap and speed of the vehicle. Other sensor parameters like focal length, spatial resolution, exposure time, and acquisition rate can be selected. The number of images required to cover the area of interest depends on all the parameters mentioned before. The influence of the image overlap is managed by the number of images per second, and it is determined using altitude and sensor configuration as shown in Equation (1).

$$
\text { Oforward }=(1-(\text { Dforward }) / \mathrm{Hw}) * 100
$$

where Oforward is the forward overlap in percent, Dforward is the distance between exposure stations $(\mathrm{m}), \mathrm{f}$ is the focal length $(\mathrm{mm}), \mathrm{H}$ is the distance from the camera projection center to the ground (m), and $\mathrm{w}$ is the width of the sensor $(\mathrm{mm})$ [17].

\begin{tabular}{|c|c|c|c|}
\hline Quality Parameters & Detail & \multicolumn{2}{|c|}{ Precision } \\
\hline Sensors Parameters & Sensor Resolution & Focal Length & Exposure Time \\
\hline Flight Parameters & Altitutde & Image Overlap & Flight Speed \\
\hline Image Parameters & Ground resolution & & er Area \\
\hline Efficiency Parameteres & Flight Time & & ssing Time \\
\hline
\end{tabular}

Figure 1. Different parameters in drone flights. The outer box represents the target variables, while the inner box shows the parameters that can be directly influenced by the mission planner and derivatives of those parameters.

The spatial resolution on the ground or ground sample distance (GSD) can be calculated using Equation (2),

$$
\operatorname{GSD}=(\mathrm{p} / \mathrm{f})^{*} \mathrm{H}
$$

where GSD is the ground sample distance $(\mathrm{cm})$, $\mathrm{p}$ is the size of a pixel $(\mathrm{mm})$ on the sensor, $\mathrm{f}$ is the focal length $(\mathrm{mm}), \mathrm{H}$ is the distance from the camera projection center to the ground $(\mathrm{cm})$. 
One of the important examples of trade-off is geometric tradeoff between altitude of the sensor and area covered during the scanning period, this is dependent on the field of view of the sensor and determines the acquisition time of the raw images. If we increase the altitude it would result in less images for the area of interest, and hence, less processing time, but increasing the altitude has direct impact on the GSD and trace detection in the imagery. On the other hand, lower altitude would lead to higher number of raw images which increases the cost of data processing but gives finer spatial resolution, which increases the probability of trace detection with increase in accuracy [18]. These tradeoffs can be used to balance the accuracy and efficiency of the hyperspectral imaging system. Even though these tradeoffs are obvious in nature, there is a lack of research and publications that explore these parameters, in order to design the system for trace detection of chemical.

This paper focuses on determining the minimum mapping size of chemicals for trace detection of chemicals used in explosives based on the constraint of altitude of the sensor. The results of the experiments performed in this next section would indicate that altitude of the sensor and minimum mapping size of chemical, which has a significant effect on accuracy when both the variables were changed by relatively large amounts. The results obtained from the experiments leave the user with a flexible range of altitude and MMS that are appropriate for different applications.

\section{Methodology}

As was mentioned above, the objective of this paper is to determine and suggest the altitude of the sensor and minimum mapping size of pure chemical for finding traces of chemicals with an accuracy above $70 \%$, as shown in conceptual Figure 2. The approach used to achieve this objective is shown in Figures 3 and 4 . The experiments were conducted in outdoor conditions and suitable altitude and minimum mapping size of the chemicals were identified using the SVM model and spectral library developed in [19]. The principal component analysis and first derivative were used to select 22 optimal wavelengths out of 144 wavelengths in the range of 400-1000 nm. These selected wavelengths showed distinct spectral features and were input to the SVM model. The algorithm selects the most suitable hyperplane on the basis of maximum margin between each class. For classification of spectral data SVM has been proved as a reliable and efficient method. In this study, SVM was performed on R studio using a radial bias function along with 10-fold cross validation, which explain the relation between the target variable and independent variable, and grid search procedure. Classification accuracy is used to assess the performances of SVM models. Chemical samples of AN, TNT and C4 were prepared in different sizes which varied from $0.25^{*} 0.25 \mathrm{~cm}$ to $1 \mathrm{~cm}^{*} 1 \mathrm{~cm}$ as shown in Figure 3 . The dimensions of the chemical samples were measured accurately using the measuring instruments and distributed over the soil, which acted as the background material. The experiment was performed on a bright sunny morning at $10 \mathrm{am}$, with sun azimuth equal to 94 , and altitude above horizon equal to 53.6. The hyperspectral sensor was placed on the vehicle at different altitude varying from $40 \mathrm{~cm}$ to $150 \mathrm{~cm}$. The speed of the vehicle is determined using the in-built software of the sensor (Cube Creator by BaySpec). The speed of the vehicle holding the sensor is suggested to user depending upon the side overlap the user has requested for the mentioned altitude. These chemical samples were placed over the soil samples and marked with different colors for accuracy assessment in later part of the experiment. The reflectance data was obtained by BaySpec OCI F hypespectral sensor, which consists of a charge coupled device that has a readout mode. The images were acquired by pushbroom scanning at 45 frames per second with a field of view of $22^{\circ}$, focal length of $16 \mathrm{~mm}$ in 144 bands with spectral resolution of $4.16 \mathrm{~nm}$ from $400-1000 \mathrm{~nm}$. Hyperspectral cube was created for the chemical samples at different heights using pushbroom scanning. To detect the traces of the chemicals, present in the hyperspectral cube, the image is converted into tiff format which goes as an input to the SVM model. The SVM model determines the traces of the chemical samples present and accuracy assessment is done for all the 
images acquired at different altitude of the sensor. The suitable altitude is selected for the next experiment where the altitude is fixed and the MMS size of the chemicals are varied. The hyperspectral cube is generated and the SVM model is used to determine how many samples of each MMS were detected correctly and incorrectly. The conceptual design of the experiment is shown in the Figure 4, which gives information on how the experiment was conducted in terms of varying the altitude of the sensor and size of the chemical samples.

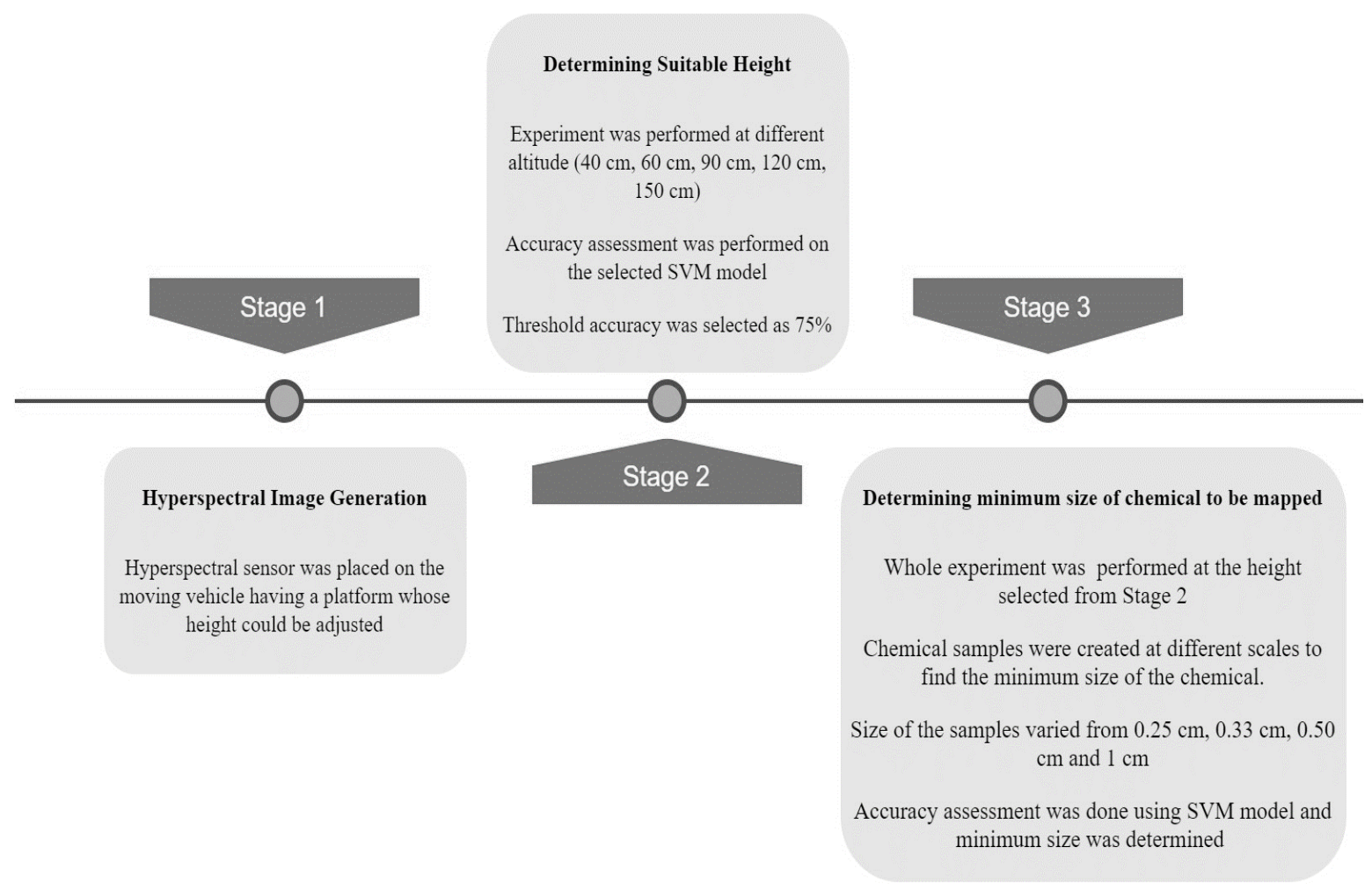

Figure 2. Conceptual design for the experiments to be conducted.

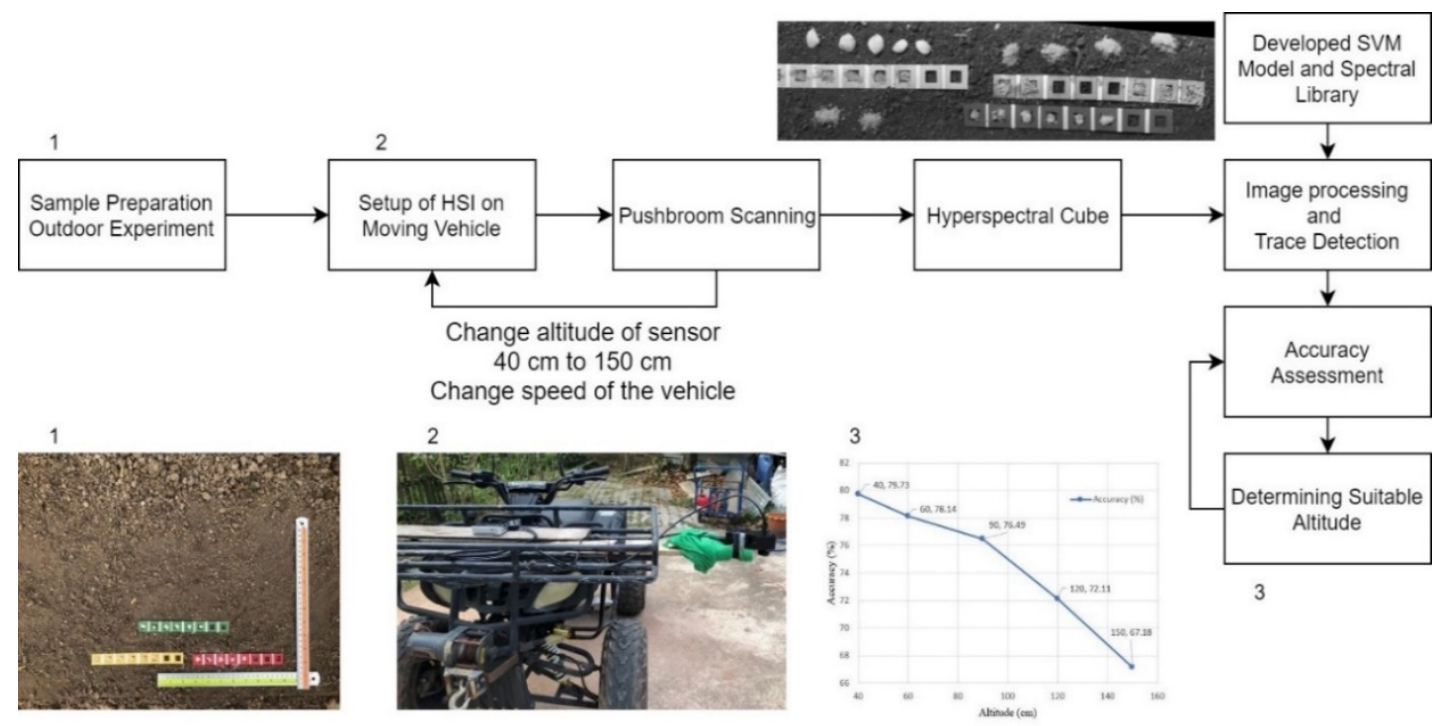

Figure 3. Steps for conducting experiment to suggest altitude of sensor in outdoor conditions. 


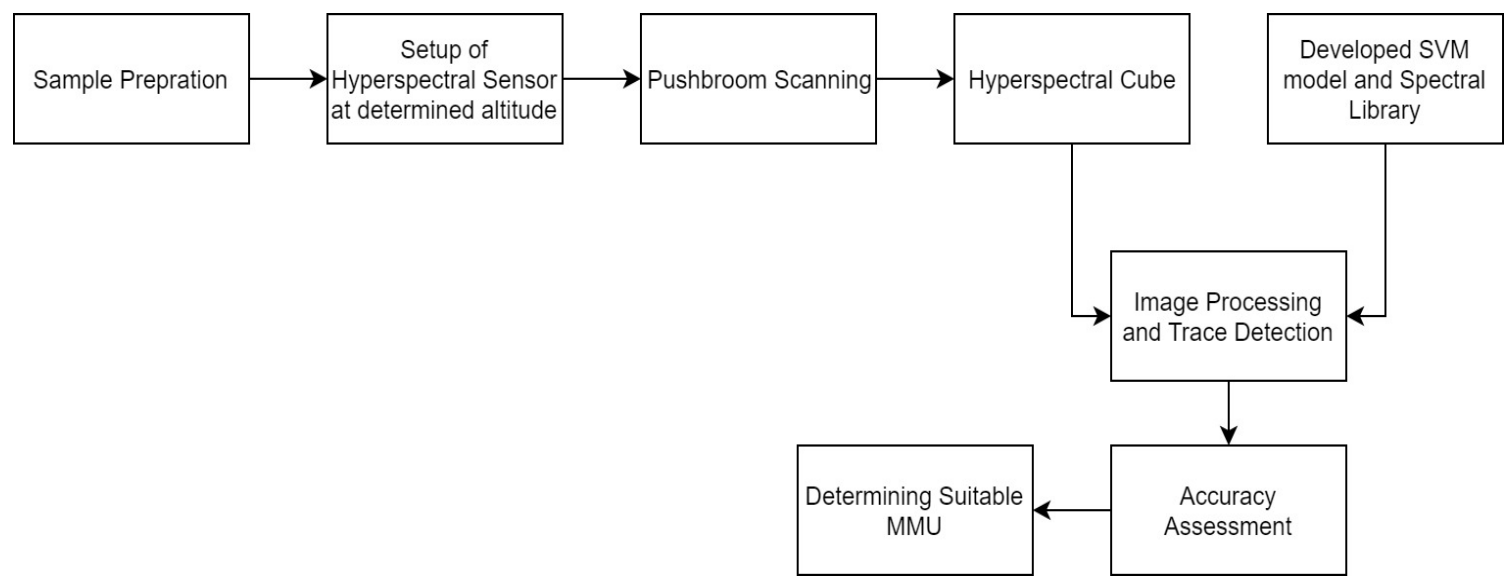

Figure 4. Flow diagram to determine suitable minimum mapping size of pure chemical used in explosives.

\section{Results}

The spatial resolution of the hyperspectral image acquired at different heights are shown in Table 1. The results of the classification accuracy for individual chemicals using the SVM model developed previously are presented. The area of soil is kept the same throughout the experiments and minimum mapping size was varied from $0.25 \mathrm{~cm}, 0.33 \mathrm{~cm}$, $0.50 \mathrm{~cm}$ and $1 \mathrm{~cm}$ as shown in Table 2. Figure 5 shows that, as the altitude of the sensor size increased, the overall percent accuracy value decreased in a linear nature. Figure 6 shows how the accuracy of the detection of chemical increase when the minimum mapping size is increased. The accuracy estimates increased gradually until the minimum mapping size reached $0.5 \mathrm{~cm}$ at which point the curve began to level off. The likely explanation for the slowing rate of increase in the accuracy estimates is that the minimum mapping size became sufficiently large that a smaller percentage of areas. From the results, an accuracy of more than $75 \%$ can be achieved when the sensor is placed at a height of $90 \mathrm{~cm}$ and similarly the chemical sample with size of $0.5 \mathrm{~cm}$ to $1 \mathrm{~cm}$ gave desirable results, whereas samples of size less than $0.5 \mathrm{~cm}$ gave low accuracy. Therefore, the suggested height for placing the sensor on the vehicle can be around $90 \mathrm{~cm}$ and the minimum mapping size can be selected as $0.5 \mathrm{~cm}$.

Table 1. Determines the accuracy of chemical at different height of sensors.

\begin{tabular}{cccc}
\hline Altitude (cm) & Spatial Resolution $(\mathbf{c m})$ & No. of Pixels & Accuracy $\mathbf{( \% )}$ \\
\hline 40 & 0.012 & 1568 & $79.73 \pm 0.02$ \\
60 & 0.018 & 784 & $78.14 \pm 0.02$ \\
90 & 0.027 & 523 & $76.49 \pm 0.03$ \\
120 & 0.036 & 392 & $72.11 \pm 0.05$ \\
150 & 0.045 & 313 & $67.18 \pm 0.04$ \\
\hline
\end{tabular}

Table 2. Determining accuracy for different sample size of chemicals.

\begin{tabular}{cccc}
\hline Sample Size (cm) & No. of Pixels & Spatial Resolution $\mathbf{( c m )}$ & Accuracy (\%) \\
\hline 1 & 38 & 0.028 & $79.00 \pm 0.02$ \\
0.5 & 19 & 0.028 & $72.77 \pm 0.04$ \\
0.33 & 13 & 0.028 & $54.94 \pm 0.04$ \\
0.25 & 9 & 0.028 & $48.69 \pm 0.10$ \\
\hline
\end{tabular}




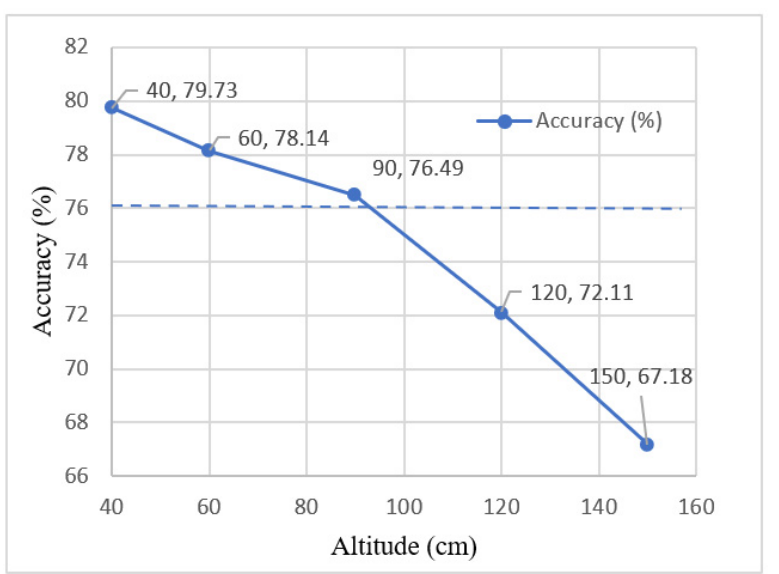

Figure 5. Accuracy of chemical at different altitude of sensors.

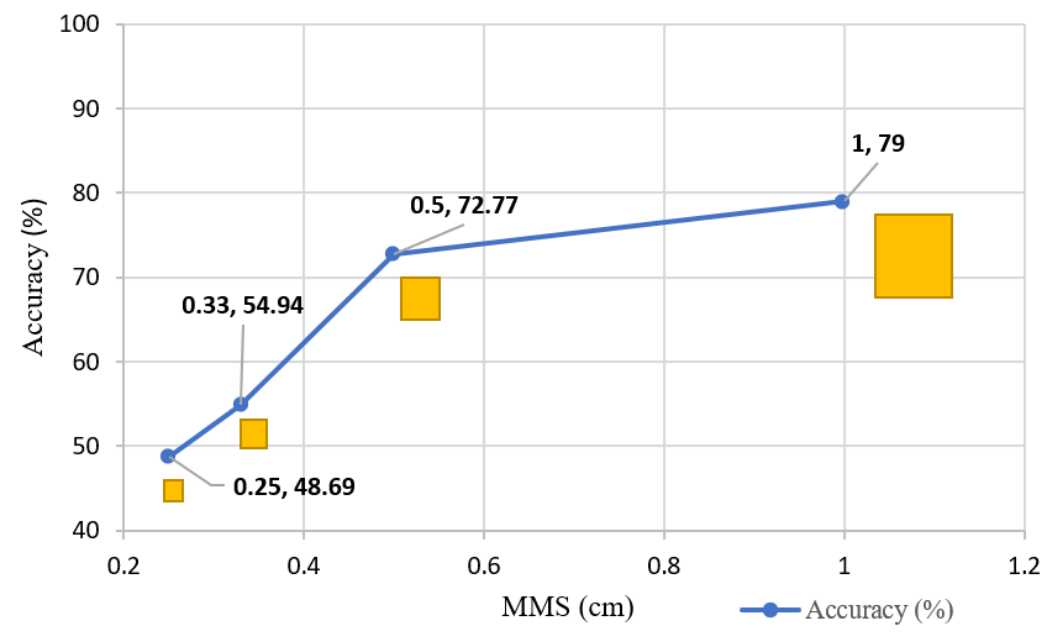

Figure 6. Mapping accuracy for different size of chemical samples.

\subsection{Influence of Image Overlap}

The influence of image overlap on image acquisition time is shown Figure 7a, and post processing time is discussed in next section. A quadratic growth was observed in acquisition time and post processing time when image overlap was increased (Figure $7 \mathrm{~b}$ ).

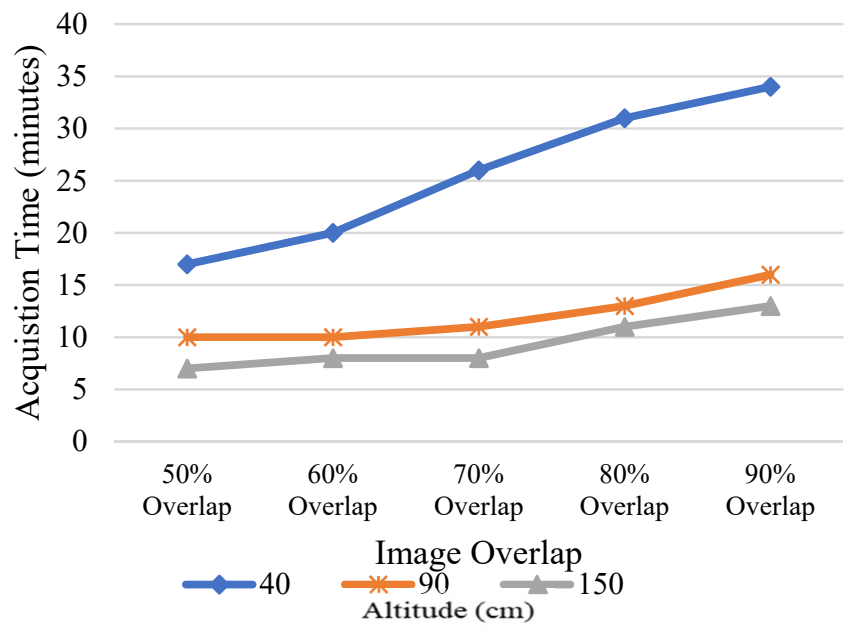

(a)

Figure 7. Cont. 


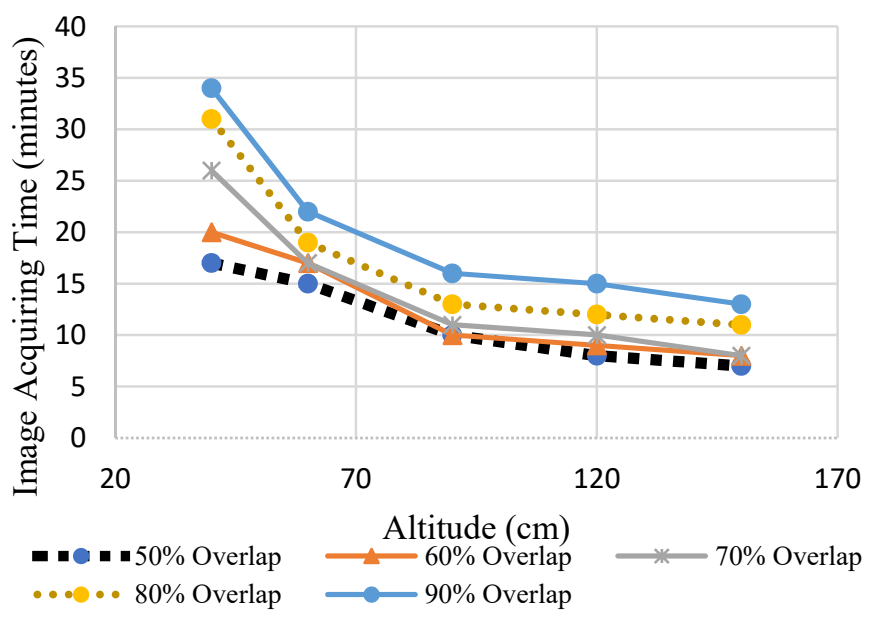

(b)

Figure 7. (a) Influence of image overlap on image acquisition time; (b) influence of altitude on image acquisition time.

Following are the results about determining the speed of the sensor for different altitude. According to the manufacture of the sensor the suggested speed is based upon the percentage of image overlap user wants. The SpecGrabber, which is the software used for controlling the sensor, and calculates the suggested speed based on the altitude, field of view, swath and consider image overlap as $70 \%$. Speed is calculated for different altitude and different overlap percentage, as shown in Table 3, and in Table 4 the suggested speed for different altitude is shown. The vehicle operator can select the speed from the Table 3 based on their requirement of overlap. Figure 8 shows the minimum, maximum and suggested speed. Table 5 discusses the flight time and number of raw images the sensor will store when scanning an area of $10 \mathrm{~m}^{2}$ at a speed of $10.5 \mathrm{~cm} / \mathrm{s}$ at different altitude.

Table 3. Determining speed $(\mathrm{cm} / \mathrm{s})$ for various overlapping $\%$ at different altitude.

\begin{tabular}{cccccc}
\hline $\begin{array}{c}\text { Altitude } \\
(\mathbf{c m})\end{array}$ & $\mathbf{5 0 \%}$ Overlap & $\mathbf{6 0 \%}$ Overlap & $\mathbf{7 0 \%}$ Overlap & $\mathbf{8 0 \%}$ Overlap & $\mathbf{9 0 \%}$ Overlap \\
\hline 40 & 7.8 & 6.2 & 4.7 & 3.1 & 1.6 \\
60 & 11.6 & 9.3 & 7.0 & 4.7 & 2.3 \\
90 & 17.5 & 14.0 & 10.5 & 7.0 & 3.5 \\
120 & 23.3 & 18.6 & 14.0 & 9.3 & 4.7 \\
50 & 29.1 & 23.3 & 17.5 & 11.6 & 5.8 \\
\hline
\end{tabular}

Table 4. Altitude and suggested speed for sensors altitude.

\begin{tabular}{cc}
\hline Altitude $(\mathrm{cm})$ & Suggested Speed $(\mathrm{cm} / \mathbf{s})$ \\
\hline 40 & 4.7 \\
60 & 7.0 \\
90 & 10.5 \\
120 & 14.0 \\
150 & 17.5 \\
\hline
\end{tabular}




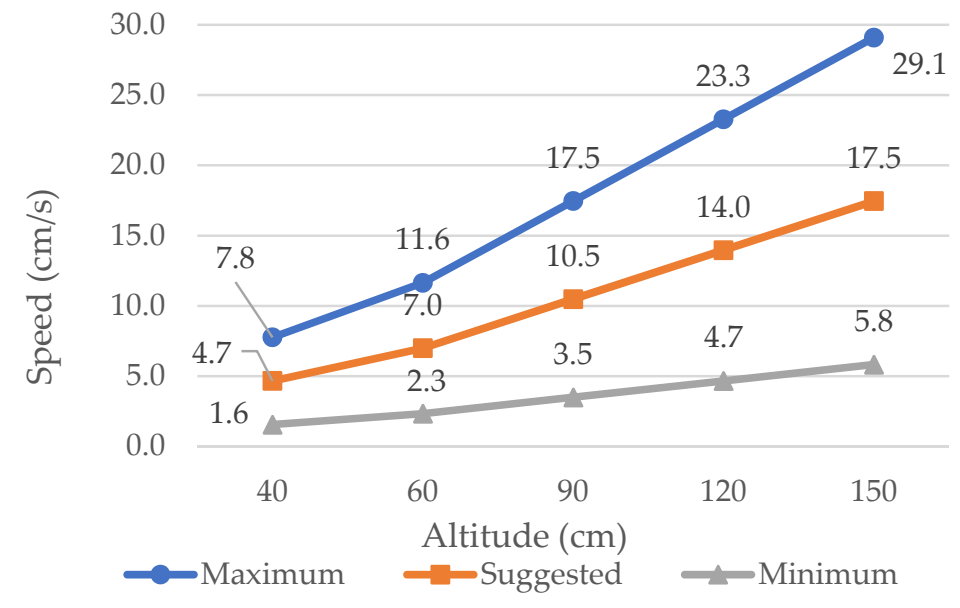

Figure 8. Minimum, suggested, and maximum speed for different altitude.

Table 5. Determining number of images, storage and flight time at $10.5 \mathrm{~cm} / \mathrm{s}$.

\begin{tabular}{ccccc}
\hline Altitude $(\mathbf{c m})$ & Speed $(\mathbf{c m} / \mathbf{s})$ & No. of Images & Storage (GB) & Flight Time (min) \\
\hline 40 & 10.5 & 1728 & 21.25 & 26 \\
60 & 10.5 & 768 & 9.45 & 17 \\
90 & 10.5 & 285 & 3.5 & 11 \\
120 & 10.5 & 195 & 2.2 & 10 \\
150 & 10.5 & 130 & 1.6 & 8 \\
\hline
\end{tabular}

\subsection{Processing Time of the Hyperspectral Cube}

The hyperspectral cube was created by processing the raw images in the Cube creator. The altitude of the sensor and image overlap percentage has the same weightage in influencing the number of raw images to be acquired by the hyperspectral imaging system. The forward overlap has an exponential impact on the processing time of the raw image and creation of hyperspectral cube. In Figure 9, a significant increase in processing time from $70 \%$ overlap is visible. While, $40 \mathrm{~cm}$ altitude has a higher processing time than $150 \mathrm{~cm}$. The resolution of the sensor has linear influence on processing time. From the observed results it can be concluded that processing time is highly dependent on number of raw images as compared to sensor resolution.

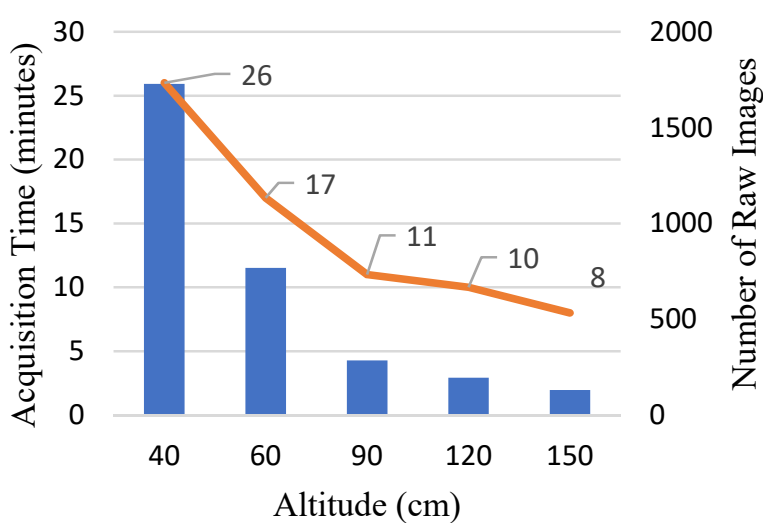

No. of Images $\quad$ Flight Time (min)

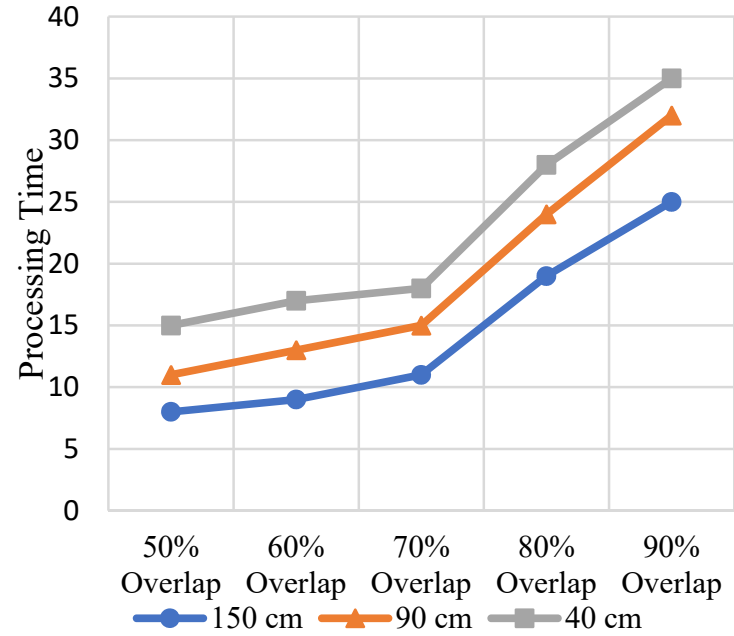

(b)

Figure 9. (a) Acquisition time for different altitude at 70\% image overlap; (b) Image processing time in minutes for different overlap percentage at $40 \mathrm{~cm}, 90 \mathrm{~cm}, 150 \mathrm{~cm}$, respectively. 


\section{Discussion}

\subsection{Influence MMS and Altitude of Sensor}

The effect of the altitude of the sensor and spatial resolution on the overall accuracy of the SVM classification model has been shown in the previous section. Based on the results obtained from different experiments, conducted in this study, it was observed that the image with a finer resolution achieved higher accuracy but at a lower altitude which might not be feasible in real applicability. While, images collected at a coarser resolution, but at a height of $150 \mathrm{~cm}$, achieved an accuracy of around $65 \%$ because the images contained traces of different chemical and soil as background. Therefore, with the increase in altitude, the dominance of the reflectance in the pixel was more from soil instead of pure chemicals, which leads to decrease in accuracy and misclassification. The misclassification error can be grouped as a pure pixel classification error or mixed pixel classification error. The mixed pixel between the soil and the chemical was the major reason for lower accuracy. To determine the suggested MMS for trace detection of chemicals the spatial resolution of the image was kept fixed and the pixels were subdivided into 0.5, 0.33 and $0.25 \mathrm{~cm}$ MMS. For studying the effect of MMS on the accuracy the image of chemical at resolution of $0.028 \mathrm{~cm}$ at an altitude of $90 \mathrm{~cm}$, consisting of 38 pixels was reduced to 19,13 and 9 pixels. As discussed earlier, the misclassification occurred due to mixed pixel where a pixel is made up of from two or more classes. This kind of error occurs often in the boundaries between two classes and is also referred to as boundary effect. From the results it can be seen and discussed that when the MMS was $1 \mathrm{~cm}$ in that case 30 pixels out of 38 pixels were correctly classified whereas the pixels around the boundary were misclassified. Similarly, for 0.5 MMS the 14 pixels out of 19 were misclassified and similarly pixels around the boundary which had dominance of soil were misclassified. However, when MMS was decreased to $0.25 \mathrm{~cm} 4$ pixels out of 9 were only classified correctly.

For practical applicability of the hyperspectral imaging system, the user needs to decide the altitude and MMS according the accuracy they want to achieve, which could be either the screening phase or critical phase. One of major factors that affects the real applicability is the amount of storage required, processing time for creation of hyperspectral cube. The speed of the vehicle mounted with sensor determines coverage area in a given time and number of raw images that would be acquired. From the experiment firstly suitable altitude was determined. A total of $90 \mathrm{~cm}$ is the suggested altitude for the user as it is easier to deploy a sensor at an altitude of $90 \mathrm{~cm}$ rather $40 \mathrm{~cm}, 60 \mathrm{~cm}$ in an automated vehicle. The coverage area or swath at $40 \mathrm{~cm}, 60 \mathrm{~cm}$ is comparatively low to $90 \mathrm{~cm}$. At $90 \mathrm{~cm}$ the user can expect an accuracy of more than $75 \%$, which would decrease if the altitude is increased to $150 \mathrm{~cm}$. While, $90-120 \mathrm{~cm}$ can be suggested altitude for the sensor to be deployed in real ground application. After determining a suitable height of $90 \mathrm{~cm}$, suitable MMS was determined $(0.5 \mathrm{~cm})$ as the accuracy achieved was more than $70 \%$ and it had minimal effect of mixed pixel problem if compared to $0.33 \mathrm{~cm}$ to $0.25 \mathrm{~cm}$, later two MMS had a very low accuracy almost equal to $50 \%$. For $90 \mathrm{~cm}$ height the speed can be varied from $3.5 \mathrm{~cm} / \mathrm{s}$ to $17.5 \mathrm{~cm} / \mathrm{s}$ depending upon the required overlapping by the user. The suggested overlap is $70 \%$, which results in a uniform stitching in the raw images for creation of hyperspectral cube. For $70 \%$ overlap, the suggested speed is $10.5 \mathrm{~cm} / \mathrm{s}$, whereas it could be $17.5 \mathrm{~cm} / \mathrm{s}$ for $50 \%$ overlap and $3.5 \mathrm{~cm} / \mathrm{s}$ for $90 \%$ overlap. For real ground conditions controlling the automated vehicle at $3.5 \mathrm{~cm} / \mathrm{s}$ is not feasible as it would a much higher time to cover the area of interest. If the vehicle is moving at a speed of $10.5 \mathrm{~cm} / \mathrm{s}$ to cover an area of $10 \mathrm{~m}^{2}$ at altitude of $90 \mathrm{~cm}$, the system would acquire 285 raw images with storage of $3.5 \mathrm{~GB}$. Whereas, if the altitude is $60 \mathrm{~cm}$, the system would acquire 768 raw images with storage of $9.45 \mathrm{~GB}$, which is more expensive and not feasible when size of the area is increased. Based on several calculation and discussions with final users, it was decided that $(90-120 \mathrm{~cm}$ ) altitude, $0.5 \mathrm{~cm}$ MMS and $10.5 \mathrm{~cm} / \mathrm{s}$ speed is highly recommended for practical usage of hyperspectral imaging system. 


\subsection{Future Opportunities}

The focus of this study was to determine the minimum size of the chemical, flight parameters like altitude, speed, image overlap, which can be controlled by the operator directly, and that would directly impact the construction of the hyperspectral cube and accuracy of the model in identifying the traces of the chemical. It was beyond the scope of the study to assess the impact of temperature, wind speed, cloud cover conditions and mass of the chemical. In the study conducted by [20] the relationship between different weather conditions and image construction has been discussed. The study shows that wind speed has a negligible impact whereas cloud cover has an influence in the quality of the image. Since weather conditions cannot be controlled, we conducted experiments in sunny conditions around 11:00 a.m., with minimum wind speeds and placed the chemicals with different areal dimensions. In this study, we created the hyperspectral cube and processing approach with only CubeCreator, which is commercial software of BaySpec. The image construction algorithm might have a different level of performance if tested on different software's in future. This software has been used and demonstrated in different studies, and thus, it has a strong baseline which can be used for comparisons.

The hyperspectral image was constructed using image to image reconstruction solutions. GNSS was not used in the process of reconstruction of the image and the whole process was dependent on the image features and position of the camera. A better image could be constructed in future using a hyperspectral sensor along with GNSS. Storage and disposal of the explosive chemicals has led to environmental issue, endangering health and safety of the public making it a major concern worldwide [21,22]. Similar HSI can also be used in the future to find sites of illegal waste disposal and leakage of hazardous chemical in industries.

\section{Conclusions}

In summary, we arrived at several conclusions with regards to the altitude of the sensor, minimum chemical size and forward overlap percentage for reconstruction of the image. To determine the suggested altitude and minimum size of the chemical that can be detected in the acceptable range of accuracy, several experiments were performed in real ground conditions. This research focused on the applicability of the proposed method in the real world. In the first set of experiments, the altitude of the sensor was varied from $40 \mathrm{~cm}$ to $150 \mathrm{~cm}$, and the accuracy of the model was determined. From the analysis, it was found that the suggested altitude should be between $90-120 \mathrm{~cm}$ above ground, and in combination with forward overlap rates of close to $70 \%$, it led to the best reconstruction detail and accuracy of the model where accuracy was more than $75 \%$. In the second set of experiments, the minimum size of the chemical sample was varied from $0.25 \mathrm{~cm}$ to $1 \mathrm{~cm}$. Accuracy of more than $70 \%$ in trace detection of chemicals with minimum chemical size of $0.5 \mathrm{~cm}$ was identified at suggested altitudes of $90-120 \mathrm{~cm}$. This accuracy of the model cannot be achieved at coarser resolution at higher altitudes since the sensor resolution had linear effect, while other parameters had a non-linear effect. The non-linear effects of forward overlap and altitude on processing time might pose a constraint on using forward overlap rates higher than $70 \%$ if processing time is the main limitation. For various altitudes, the suggested speed of the vehicle were calculated. Therefore, to implement hyperspectral imaging system on the unarmed vehicle for real application, the suggested altitude and speed of the sensor should be around $90 \mathrm{~cm}$ and $10.5 \mathrm{~cm} / \mathrm{s}$ at which the detection limit would be equal or more than $0.5 \mathrm{~cm}$ with accuracy greater than $70 \%$.

Author Contributions: S.C., S.N. and T.N. contributed to the overall study design and supervised all research. S.C. finished the experiment, including the sample preparation and hyperspectral image acquisition. S.C., S.N. and T.N. contributed to the data analysis and the manuscripts. All authors have read and agreed to the published version of the manuscript.

Funding: This research received no external funding. 
Acknowledgments: The authors thank and acknowledge Foundation of Innovation for Happiness, Thailand for providing resources and support throughout the project.

Conflicts of Interest: The authors declare no conflict of interest.

\section{References}

1. Marshall, M.; Oxley, J. Aspects of Explosives Detection; Yinon, J., Ed.; Elsevier: Amsterdam, The Netherlands, 2009.

2. Golightly, R.S.; Doering, W.E.; Natan, M.J. Surface-enhanced Ramanspectroscopy and homelandsecurity: A perfect match? ACSNano 2009, 3, 2859-2869.

3. Nuntawong, N.; Eiamchai, P.; Limwichean, S.; Wong-ek, B.; Horprathum, M.; Patthanasettakul, V.; Leelapojanapom, A.; Nakngoenthong, S.; Chindaudom, P. Trace detection of perchlorate in industrial -grade emulsion explosive with portable surface-enhanced Raman spectroscopy. Forensic Sci. Int. 2013, 233, 174-178. [CrossRef] [PubMed]

4. Martín-Alberca, C.; García-Ruiz, C. Analytical techniques for the analysis of consumer fireworks. TrAC Trends Anal. Chem. 2014, 56, 27-36. [CrossRef]

5. de la Ossa, M.A.F.; Amigo, J.M.; García-Ruiz, C. Detection of residues from explosive manipulation by near infrared hyperspectral imaging: A promising forensictool. Forensic Sci. Int. 2014, 242, 228-235.

6. Hakonen, A.; Andersson, P.O.; Schmidt, M.S.; Rindzevicius, T.; Käll, M. Explosive and chemical threat detection by surfaceenhanced Raman scattering: Are- view. Anal. Chim. Acta 2015, 893, 1-13. [CrossRef] [PubMed]

7. Laska, P.R. Bombs, IEDs, and Explosives: Identification, Investigation, and Disposal Techniques; CRC Press Taylor \& FrancisGroup: Boca Raton, FL, USA, 2016.

8. Rose, A.; Zhu, Z.; Madigan, C.F.; Swager, T.M.; Bulovic, V. Sensitivity gains in chemosensing by lasing action in organic polymers. Nature 2005, 434, 876-879. [CrossRef] [PubMed]

9. Germain, M.; Knapp, M. Optical explosives detection: From color changes to fluorescence turn-on. Chem. Soc. Rev. 2009, 38 , 2543. [CrossRef] [PubMed]

10. Elbasuney, S.; El-Sherif, A.F. Complete spectroscopic picture of concealed explosives: Laser induced Raman versus infrared. TrAC Trends Anal. Chem. 2016, 85, 34-41. [CrossRef]

11. El-Sharkawy, Y.H.; Elbasuney, S. Novel laser induced photoacoustic spectroscopy for instantaneous trace detection of explosive materials. Forensic Sci. Int. 2017, 277, 215-222. [CrossRef] [PubMed]

12. Garcia, J.E.; Girard, M.B.; Kasumovic, M.; Petersen, P.; Wilksch, P.A.; Dyer, A.G. Differentiating Biological Colours with Few and Many Sensors: Spectral Reconstruction with RGB and Hyperspectral Cameras. PLoS ONE 2015, 10, e0125817. [CrossRef] [PubMed]

13. Smekens, J.-F.; Gouhier, M. Observation of SO2 degassing at Stromboli volcano using a hyperspectral thermal infrared imager. J. Volcanol. Geotherm. Res. 2018, 356, 75-89. [CrossRef]

14. Villa, A.; Chanussot, J.; Benediktsson, J.A.; Jutten, C. Spectral Unmixing for the Classification of Hyperspectral Images at a Finer Spatial Resolution. IEEE J. Sel. Top. Signal. Process. 2010, 5, 521-533. [CrossRef]

15. González, C.; Resano, J.; Mozos, D.; Plaza, A.; Valencia, D. FPGA Implementation of the Pixel Purity Index Algorithm for Remotely Sensed Hyperspectral Image Analysis. EURASIP J. Adv. Signal. Process. 2010, 2010, 969806. [CrossRef]

16. Xiangyun, X.; Gertner, G.; Wang, G.; Anderson, B. Optimal sampling scheme for estimation landscape mapping of vegetation cover. Landsc. Ecol. 2005, 20, 375-387.

17. Falkner, E.; Morgan, D. Aerial Mapping: Methods and Applications, 2nd ed.; CRC Press: Boca Raton, FL, USA, 2002.

18. Whitehead, K.; Hugenholtz, C.H. Remote sensing of the environment with small unmanned aircraft systems (UASs), part 1: A review of progress and challenges. J. Unmanned Veh. Syst. 2014, 2, 69-85. [CrossRef]

19. Chaudhary, S.; Ninsawat, S.; Nakamura, T. Non-Destructive Trace Detection of Explosives Using Pushbroom Scanning Hyperspectral Imaging System. Sensors 2018, 19, 97. [CrossRef] [PubMed]

20. Dandois, J.P.; Ellis, E.C. High spatial resolution three-dimensional mapping of vegetation spectral dynamics using computer vision. Remote Sens. Environ. 2013, 136, 259-276. [CrossRef]

21. Che, Y.; Gross, D.E.; Huang, H.; Yang, D.; Yang, X.; Discekici, E.; Xue, Z.; Zhao, H.; Moore, J.S.; Zang, L. Diffusion-Controlled Detection of Trinitrotoluene: Interior Nanoporous Structure and Low Highest Occupied Molecular Orbital Level of Building Blocks Enhance Selectivity and Sensitivity. J. Am. Chem. Soc. 2012, 134, 4978-4982. [CrossRef] [PubMed]

22. Sulzer, P.; Petersson, F.; Agarwal, B.; Becker, K.H.; Jurschik, S.; Mark, T.D.; Perry, D.; Watts, P.; Mayhew, C.A. Proton Transfer Reaction Mass Spectrometry and the Unambiguous Real-Time Detection of 2,4,6 Trinitrotoluene. Anal. Chem. 2012, 84, 4161-4166. [CrossRef] [PubMed] 